\title{
Immunohistochemical Study of CK 5/6 in Benign and Malignant Breast Lesions
}

\author{
${ }^{1}$ Ankita Das, ${ }^{2}$ Rashmi MV
}

\begin{abstract}
Introduction: Breast lesions are very common diseases worldwide. Benign breast disease (BBD) accounting for most breast problem in females. Carcinoma breast is one of the most common human neoplasms rapidly replacing cancer of cervix as the most important leading site of cancer in most urban populations of India. A panel of antibodies, improved antigen retrieval techniques have all contributed to the use of immunohistochemistry $(\mathrm{IHC})$ in solving diagnostic problems in breast pathology.
\end{abstract}

Materials and methods: This is a cross-sectional study done in a hospital in Tumkur, Karnataka for 1.5 years. Total of 41 benign and 41 malignant biopsies of breast neoplasm from female patients was studied. All breast masses were analyzed systematically by clinical history, physical examination, histopathological examination along with the immunohistochemical study of CK 5/6. All collected data were entered into a master sheet and fed into computer software for statistical analysis using Pearson's Chi-square test.

Results: In our study patients belonged to the age group of $15-70$ years. Benign lesions were common in the age group of 21-30 years, and malignant lesions were common in the age group of 51-60 years. The most frequent benign neoplasm was a fibroadenoma (74\%) and in malignancy, infiltrating ducta carcinoma-NOS (85\%). Most of the malignant cases were of size T2 (76\%) and grade II (66\%) and without any lymph node metastatic deposits (83\%). All benign breast lesions showed positive expression for CK 5/6; the staining index of benign lesions varied from 5-9. Fibroadenomas showed stain index range of $6-8$. Highest stain index of 9 was seen in fibrocystic with ductal epithelial hyperplasia. In malignant lesions, all cases were negative for CK 5/6 expression except 3 IDC-NOS cases which were grade III (poorly-differentiated) and showed weak CK 5/6 expression with stain index of 2.There was a statistically significant association between $\mathrm{CK} 5 / 6$ stain index with grading and lymph node metastasis.

Conclusion: Immunohistochemistry (IHC) is an integral part of the pathology. Although hematoxylin and eosin (H\&E) stain remain the gold standard method for diagnosis, IHC provides useful vital information in grey zone cases. CK $5 / 6$, as a component of panels along with AE1/ AE3 and myoepithelial markers, help to differentiate benign and malignant breast lesions in cases of interobserver variability. Grade III breast carcinoma

\footnotetext{
${ }^{1}$ Senior resident, ${ }^{2}$ Professor

1,2 Department of Pathology, Sri Siddhartha Medical College and Research Centre, Tumkur, Karnataka, India

Corresponding Author: Ankita Das, Senior Resident, Department of Pathology, Sri Siddhartha Medical College and Research Centre, Tumkur, Karnataka, India, e-mail: drankitadas1990@gmail.com
}

cases, if positive for CK 5/6, imply a 'basal-like' molecular phenotype and signify a poor prognosis. These tumors require aggressive intervention. CK 5/6 can help provide prognostic information and better treatment modalities.

Keywords: Benign breast disease, Breast carcinoma, CK 5/6.

How to cite this article: Das A, Rashmi MV. Immunohistochemical Study of $\mathrm{Ck} 5 / 6$ in Benign and Malignant Breast Lesions. J Med Sci 2018;4(4):95-102.

Source of support: Nil

Conflict of interest: None

\section{INTRODUCTION}

A number of cyclical hormonal changes takes place in a woman's breast throughout her reproductive life. Breast diseases are showing a rising trend worldwide. A wide spectrum of disorders ranging from the self-limiting inflammatory lesion, benign breast lesion to life-threatening invasive carcinoma arises from the breast.

Benign breast diseases (BBD) comprise a wide range of disorders from developmental abnormality, inflammatory lesions, epithelial and stromal proliferation to the neoplasm. BBD is the most common cause of breast problem among women with a prevalence rate of $68 \%$ among all breast lesions in India. It starts to rise in the second decade of life, peaks in the third decade and decline thereafter whereas the incidence of breast carcinoma rises as age advances. Because of its high incidence and cancerous potential of certain histological types, BBD needs to be given utmost attention. ${ }^{1}$

A BBD is more frequent than malignant ones. Thirty percent of women suffering from benign breast diseases require treatment at some point in their lives. ${ }^{2}$

Among all female cancers, breast cancer is most common worldwide representing almost a quarter $(25 \%)$ of all. Cancer breasts constitute $14.3-30 \%$ of all cancers in women (as per the data collected from the hospitalbased cancer registry). The data from the report of the project on "Development of an Atlas of Cancer in India" shows that in Hospital Based Cancer Registry breast cancer is leading in Mumbai and Trivandrum. The second leading states include Bengaluru and Chennai. ${ }^{3}$ As per the statistics reported in GLOBACON, WHO 2012 (IARC) in India 1,44,937 women were detected with whereas 70,218 women died of breast carcinoma. ${ }^{4}$ The breast carcinoma reported incidence rates from the National 
Cancer Registry Program (NCRP) data indicate the percentage of carcinoma cases compared to total cases over time in Bengaluru and Chennai have increased. ${ }^{4}$ Also by 2010-2020 NCRP indicate that there will be more than 100,000 estimated breast cancer cases annually in India based on NCRP data in Bengaluru. ${ }^{5}$ In most urban populations of India, carcinoma breast has a higher incidence compared to carcinoma cervix. ${ }^{3}$ Several etiological factors: age, family history, genetics, alcohol, diet, obesity, sedentary lifestyle, endocrine factors are implicated. Current management of carcinoma of the breast is multimodality treatment which includes surgery, radioherapy, and chemotherapy and hormone therapy.

A triple breast assessment is done by clinical examination, radiological imaging (USG or mammography) and image result for fine needle aspiration cytology (FNAC) or core needle biopsy, allows the majority of patients with discrete BBDs to arrive at a diagnosis. An early diagnosis and treatment plan helps in reducing unnecessary anxiety about breast carcinoma and those with an increased risk of malignancy (atypical hyperplasia) are given prompt treatment thus improving prognosis. ${ }^{2}$ Diagnosis of benign and malignant epithelial lesions is done using hematoxylin-eosin microscopic sections alone. However in grey zone cases such as distinguishing usual ductal hyperplasia from atypical ductal hyperplasia/ductal carcinoma in situ (DCIS), diagnosing lobular vs. ductal or basal versus luminal, to identify true micro invasion, to improve sentinel lymph node staging, to localize metastatic carcinoma of unknown primary site as of breast origin, use of immunohistochemical stains are of great help in these difficult areas particularly when diagnosis can significantly impact on management and prognosis. $^{6}$

Based on antigen-antibody recognition immunohistochemistry is used for localizing specific antigens in tissue/cells. Cytokeratin an intermediate filament protein indicate epithelial cell type, state of tissue growth, differentiation, functional status and used for fingerprinting of various carcinomas. The normal breast tissue is composed of luminal cells that express CK 8/18, CK 7, and CK 19. The basal/ myoepithelial cells express CK 5/6, CK 14, CK 17 and SMA. All benign breast lesions except lactating adenoma show positivity with CK 5/6.

The treatment and prognosis of DCIS and UDH differ significantly, but the morphology has overlapping features giving rise to interobserver variability. Immunostaining with CK 5/6 aid to reach a definitive diagnosis. ${ }^{7}$

This antibody is applied very frequently to help differentiate invasive from noninvasive lesions, e.g. radial scar from grade I invasive carcinoma, microglandular adenosis from tubular carcinoma, intraductal papilloma vs. papillary intraductal carcinoma. Grade III breast carcinomas positive for CK 5/6 imply basal-like molecular phenotype and require aggressive intervention. Immunohistochemical staining with $C K 5 / 6$, as a component of panels along with AE1/AE3 and myoepithelial markers: smooth muscle actin, smooth muscle myosin heavy chain, p63 help differentiate benign and malignant breast lesion in case of interobserver variability. Histomorphological study of breast carcinomas with CK 5/6 IHC along with H-E stained section will be helpful in differential diagnosis of preinvasive breast diseases thus becoming useful in routine usage. ${ }^{7}$

\section{MATERIALS AND METHODS}

This is a cross-sectional study done in a hospital in Tumkur, Karnataka for a period of 1.5 years. Total of 41 benign and 41 malignant biopsies of breast neoplasm from female patients were studied. Non-neoplastic lesions were excluded from the study.

After taking consent, detailed history of the patient including past history and family history were taken. Thorough physical examination of the patient was done to find out regarding position, size, shape, mobility, consistency of palpable breast mass, to know about skin changes over breast if any puckering, dimpling, nipple retraction, discharge, peau-d-orange appearance or any lymph node metastasis.

Biopsy is done to confirm the diagnosis. Biopsy specimens were received in $10 \%$ formalin. In cases where mastectomy was done whole breast mass was received and grossing done as per standard procedures.

Specimens were then systematically examined. Gross details regarding site, size, surface, capsule, margins, calcification, consistency, necrosis, tumor area, and lymph node involvement examined. Representative tissue section processed routinely by paraffin section for light microscopy.

Benign tumors include: fibroadenoma, phyllodes tumor, fibrocystic disease, papillomas, tubular adenoma, lactating adenoma, epithelial hyperplasia.

Malignant include atypical ductal hyperplasia, atypical lobular hyperplasia, carcinoma-in-situ (DCIS, LCIS), infiltrating carcinomas (IDC-NOS, ILC, tubular carcinoma, medullary carcinoma, mucinous carcinoma, invasive papillary carcinoma, Invasive micropapillary carcinoma, apocrine carcinoma, secretory carcinoma, etc).

After properly classifying the tumor histopathologically, paraffin blocks of the representative section were processed for immunohistochemistry of CK 5/6. Controls were run simultaneously. CK 5/6 expression was evaluated on basis of extent and intensity of immunohistochemical expression in cytoplasm alone or along with membrane stain by microscopy using a scale from 0 to $3 .{ }^{8}$ 


\section{Intensity}

0 No staining

$1+$ Weak staining,

$2+$ Moderate staining, $2+10-50 \%$ immunopositive cells

$3+$ Strong staining, $\quad 3+>50 \%$ immunopositive cells

\section{Statistical Analysis}

All data collected were entered into a master sheet and then fed into computer software for statistical analysis using Pearson's Chi-square test.

\section{RESULTS}

During the period of study of 1.5 years, a total of 41 benign and 41 malignant biopsies of breast neoplasm from female patients were studied in a hospital in Tumkur, Karnataka. CK 5/6 IHC was performed in all cases. In our study, the patients belonged to the age group of 15-70 years with a median of 37.5 years. Benign lesions were common in the age group of 21-30 years and malignant lesions were common in the age group of 51-60 years. The most common benign neoplasm found in our study was fibroadenoma (74\%) and in malignancy, infiltrating ductal carcinoma-Nos (85\%). In the present study, the highest number of 31 cases were of size T2 $(>2 \mathrm{~cm}$ and $<5 \mathrm{~cm})$. The majority were grade II tumors in 27 patients $(66 \%)$ followed by grade I in 11 patients (27\%). Only grade III was found in 3 patients (7\%). A total of 7 cases showed positive for metastatic deposit in the lymph node. Rest were negative. All benign breast lesions showed positive expression for CK 5/6; the staining index of benign lesions varied from 5-9 (Figs 1 and 2). Highest stain index 9 was seen in fibrocystic with ductal epithelial hyperplasia. Most fibroadenomas showed a stain index of 6 (Figs 3 and 4). Benign phyllodes showed stain index of 5 (Figs 5 and 6, Table 1). Three out of 35 IDC-NOS cases were grade III (poorly-differentiated) and showed weak CK

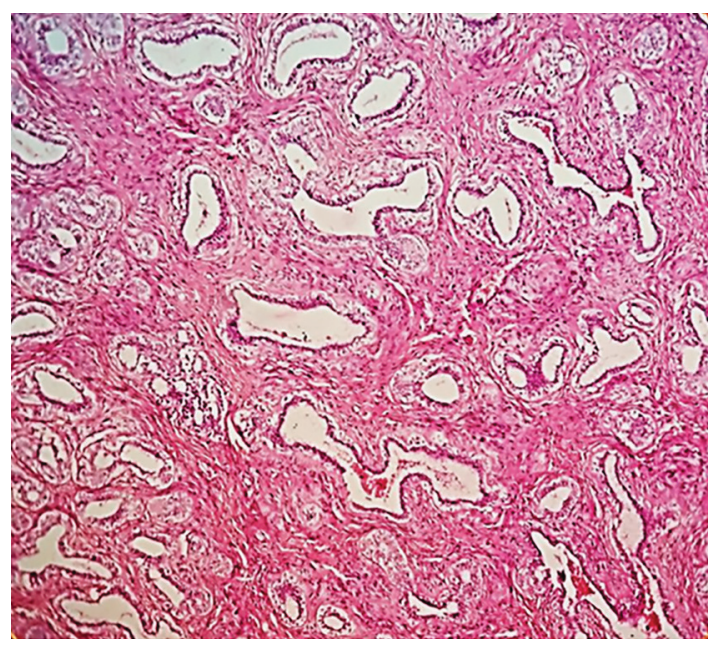

Fig. 1: Tubular adenoma ( $\mathrm{H}$ and $\mathrm{E} 10 \mathrm{X})$
$5 / 6$ expression with stain index of 2 (Fig. 7). Rest of the cases showed loss of CK 5/6 expression (Figs 8 and 15, Table 2).

In the present study 28 cases of size T2 $(>2 \mathrm{~cm}$ and $<5 \mathrm{~cm}$ ) showed CK 5/6 stain index 0 .

On applying the Chi-square test, $p=0.593$ obtained which turned out to be insignificant.

A total of 3 grade III (poorly differentiated) cases showed weak CK5/6 stain index of 2. The significant $p=0.0005$ value was obtained.

A total of 7 cases showed positive for metastatic deposit in lymph node. Out of 7, only 3 cases with positive lymph node deposits showed stain index of 2 . On applying the Chi-square test, $p=0.003$ obtained which is significant.

\section{DISCUSSION}

The present study was undertaken to study the immunohistochemistry of CK 5/6 performed on 82 breast biopsy specimens. The sample size consisted of 41 benign and 41 malignant lesions. In our study, benign lesions were commonest in the age group of 21-30 years, and malignant lesions were the wost common in 51-60 years. Past studies have shown that benign breast lesions begin to rise during the 2 nd decade of life and peaks in the 4th decade. Throughout a woman's reproductive life both epithelial and stromal elements of breast lobule are under hormonal control. Any interference with these close interactions results in conditions grouped under benign breast disease. Also repeated development and involutional changes of menstruation, pregnancies are responsible for minor aberrations.

Kapoor et al. observed malignant lesions were common in the 4 th decade followed by 5 th, 6 th, 7 th decade. ${ }^{9}$ Mudholkar observed in his study, the age range for malignant breast neoplasm was 27-80 years; maximum cases were found in the 5 th decade. ${ }^{10}$ The

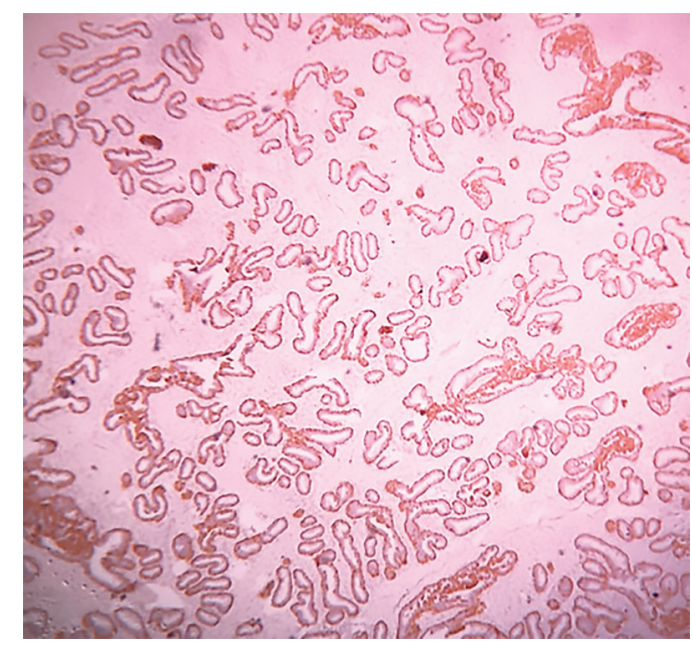

Fig. 2: Tubular adenoma CK 5/6 stain index 8 (10X) 


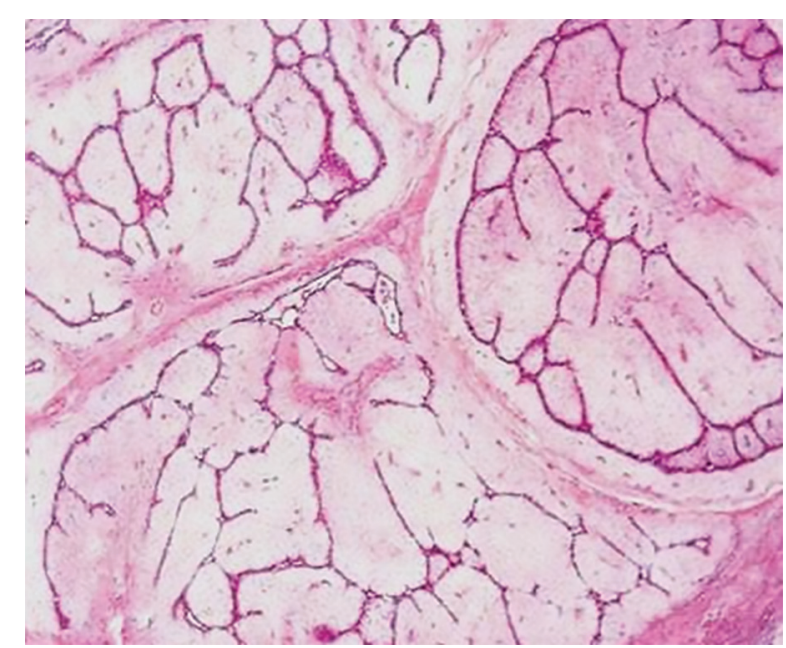

Fig. 3: Fibroadenoma (H and E 10X)

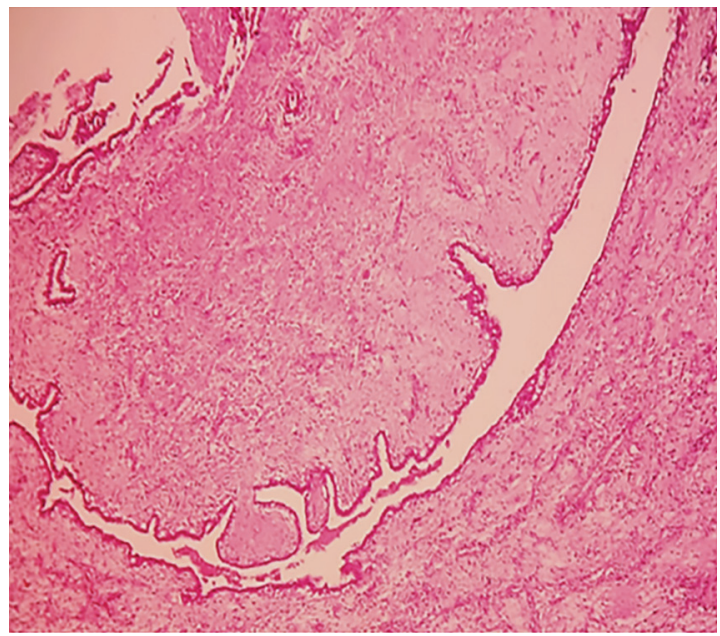

Fig. 5: Benign phyllodes (H and E $10 \mathrm{x}$ )

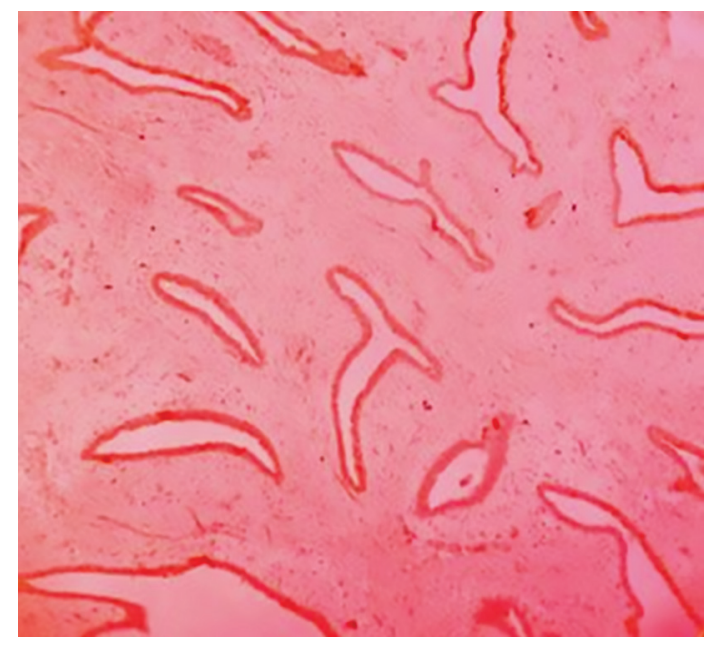

Fig. 4: Fibroadenoma CK 5/6 positive stain index 6 (10X)

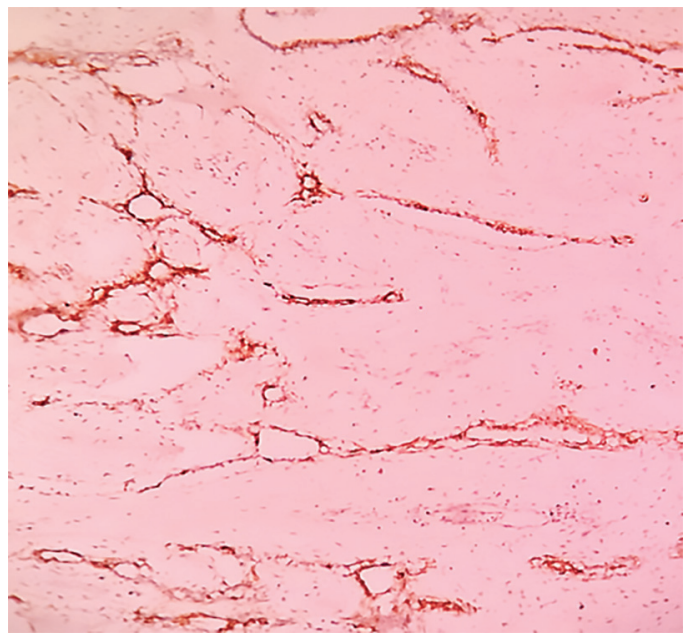

Fig. 6: Benign phyllodes CK 5/6 positive stain index 5 (10 x)

Table1: Correlation between number of benign lesions and CK5/6 stain index positivity

\begin{tabular}{lllll}
\hline Benign & $\begin{array}{l}\text { No. of } \\
\text { cases }\end{array}$ & $\begin{array}{l}\text { Age } \\
\text { (year) }\end{array}$ & $\begin{array}{l}\text { CK 5/6 positive } \\
\text { cases }\end{array}$ & No. of cases and CK 5/6 stain index \\
\hline Fibroadenoma & 30 & $17-40$ & 30 & 25 Cases SI-6 4 cases SI-7 1 case SI-8 \\
Benign phyllodes & 05 & $37-60$ & 05 & All cases SI-5 \\
Fibrocystic with ductal epithelial hyperplasia & 02 & $40-58$ & 02 & All cases SI-9 \\
Fibrocystic & 02 & $35-48$ & 02 & 1 Case SI-6 1 case SI-7 \\
Florid ductal hyperplasia & 01 & 43 & 01 & 1 Case SI-8 \\
Tubular adenoma & 01 & 15 & 01 & 1 Case SI-8 \\
\hline
\end{tabular}

incidence of breast cancer increases as age advances peaking at 70-80 years. Late menopause results in prolonged exposure to estrogens that increase risk. With increasing age breast tissue gets replaced by adipose tissue, fibrous connective tissue increase in density. Women with very dense breasts have an increased risk of carcinoma. Delayed involution and mammographic density result in accumulation of growth factors and hormones that cause excess cell division and damage to cells resulting in high breast cancer incidence. ${ }^{11}$ The risk for breast cancer also rises with increasing severity of BBD including hyperplasia with moderate to marked atypia in premenopausal women. Thus the present study is in concordance with the studies available in the literature.

The most common benign neoplasm in our study was a fibroadenoma (74\%) in agreement with other available literature. Since they arise from lobules, they are seen predominantly in $15-25$ years age group. Fibroadenomas also represent "Aberrations of normal development and involution". A direct association has been noted between oral contraceptive usage before 20 years age and risk of fibroadenoma. Mudholkar reported the highest incidence 


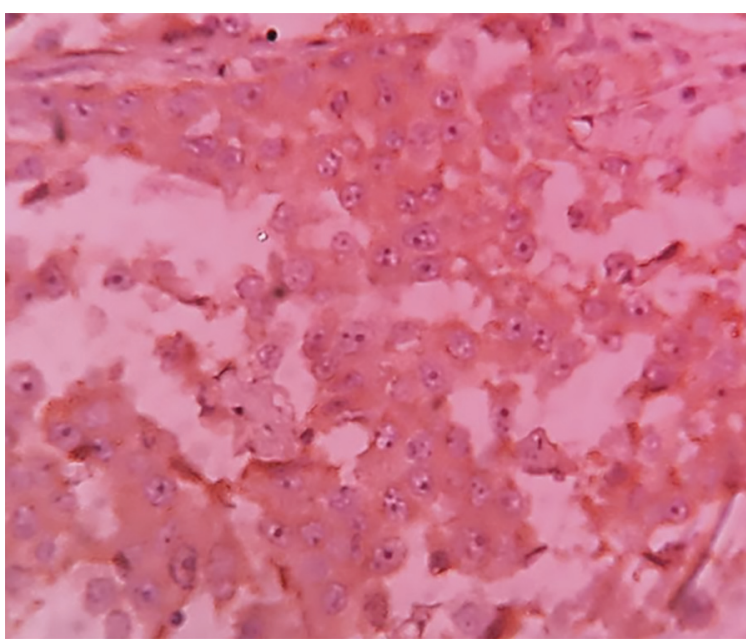

Fig 7: IDC-NOS, grade III, CK 5/6 weak positive expression stain index $2(40 x)$

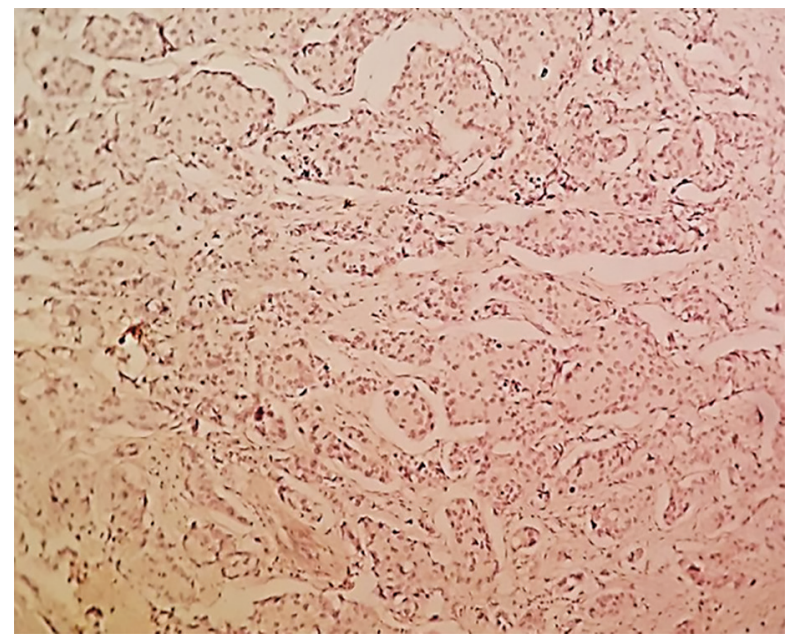

Fig 9: IDC-NOS, grade I, CK 5/6 negative score 0 (10x)

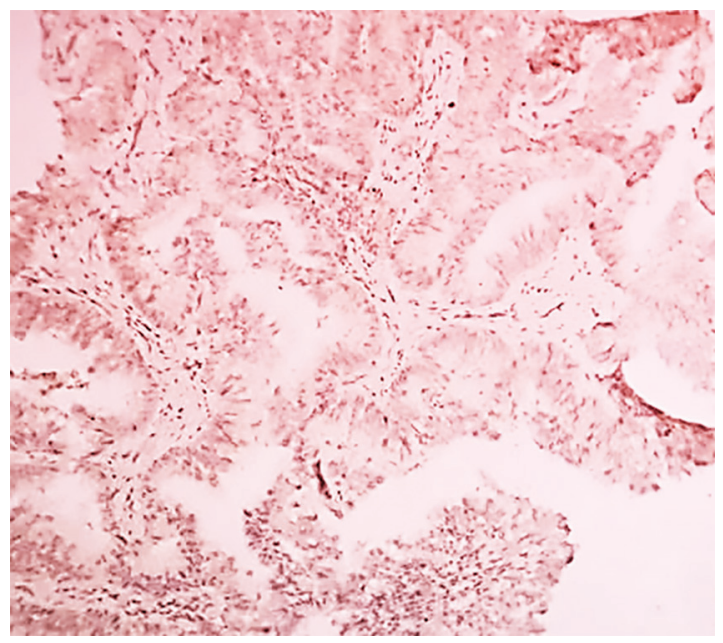

Fig. 11: Invasive papillary carcinoma, grade II, CK 5/6 negative stain index $0(10 \mathrm{X})$

of fibroadenoma $87 \%$ which could be attributed to his study of 252 cases of breast neoplasm done for a long period of 5 years.

In malignancy, commonest neoplasm was infiltrating ductal carcinoma-NOS (85\%). The findings in our study

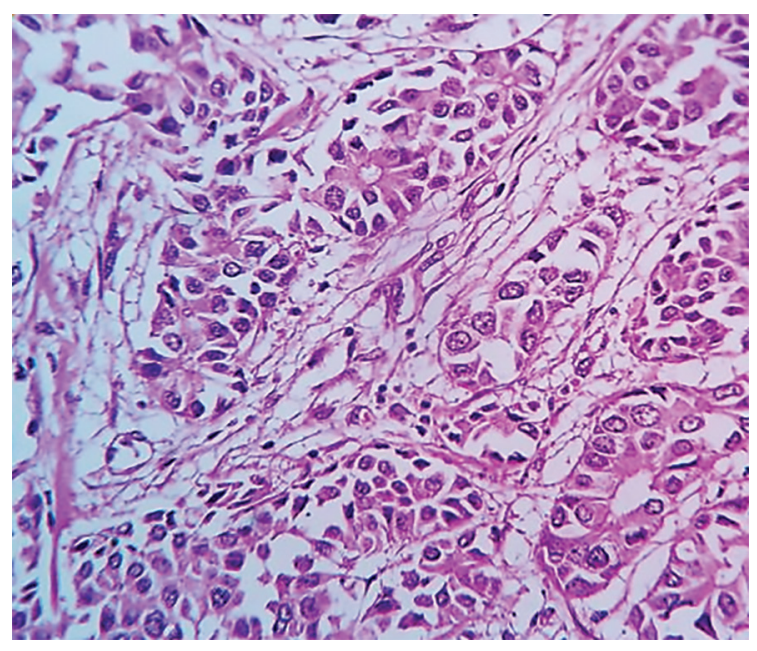

Fig 8: IDC-NOS, grade I (H and E 40x)

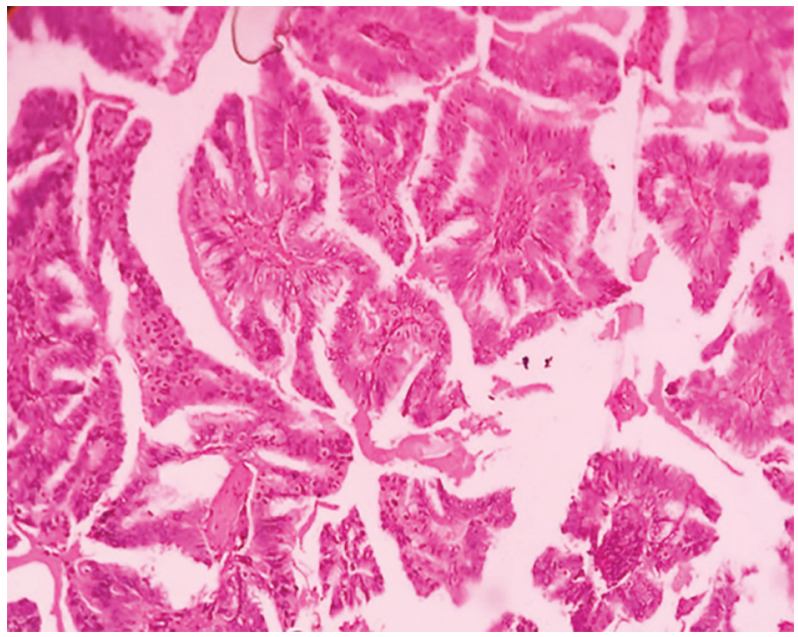

Fig 10: Invasive papillary carcinoma, grade II (H and E 10x)

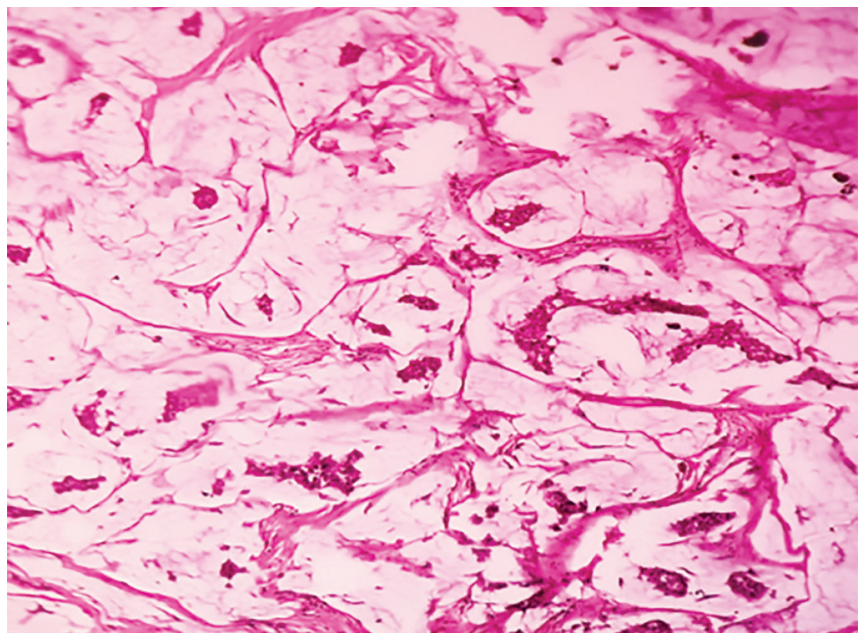

Fig. 12: Mucinous carcinoma, grade II (H and E 10)

correlated with others like Mudholkar et al., Bhalla et al., Kapoor et al.

In the present study maximum number of cases showed a tumor size of more than $2 \mathrm{~cm}$ and less than $5 \mathrm{~cm}$ (T2). A possible reason for the larger size of the 


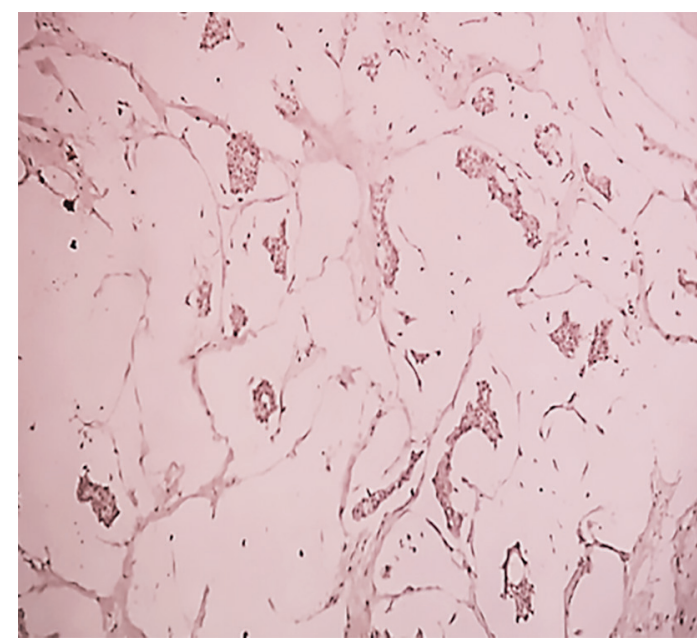

Fig. 13: Mucinous carcinoma, grade II, CK5/6 negative stain index 0 (10X)

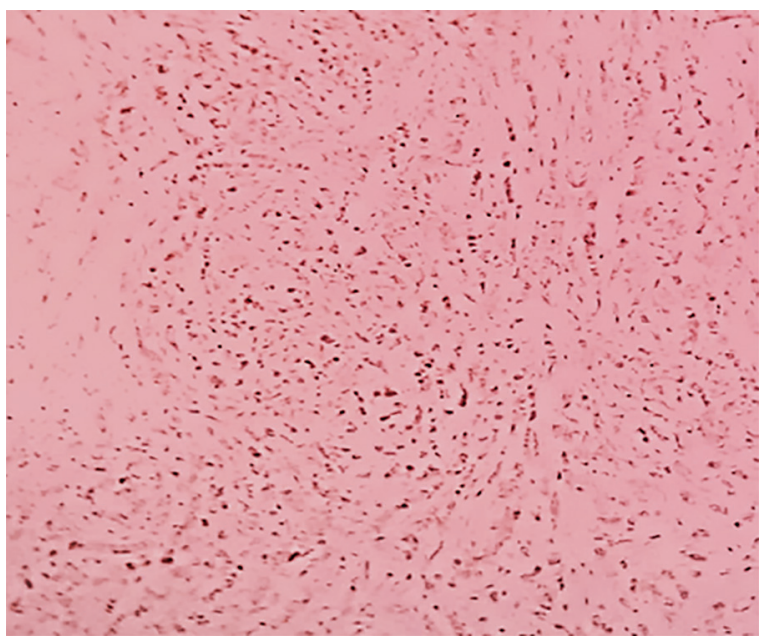

Fig. 15: Invasive lobular carcinoma, grade II CK5/6 negative stain index 0 (10X)

tumor is that this study was conducted in our hospital which is situated in a rural part of Karnataka where patients come at a later stage of the disease. Gross size of the tumor is an important prognostic factor in breast carcinoma, and with increasing size of the tumor there is an increased incidence of axillary lymph node metastases and decreased survival.

In the present study majority were grade II tumors $(66 \%)$. Only 3 cases were grade III $(7 \%)$ whi ch correlated with other studies. Our study showed a higher percentage of grade II tumors. A possible reason for this is patients presenting at a later stage of disease thus underlying the importance of regular breast self-examination. High-grade tumors significantly increase the frequency of lymph node metastases, developing more systemic recurrences, and bad prognosis. ${ }^{12}$

In our study $83 \%$ cases were negative for lymph node metastatic deposits; it may be due to the gross size of the tumor. Most of the cases in our study are of size T2. The incidence of axillary lymph node metastasis and decreased chances of survival is proportional with

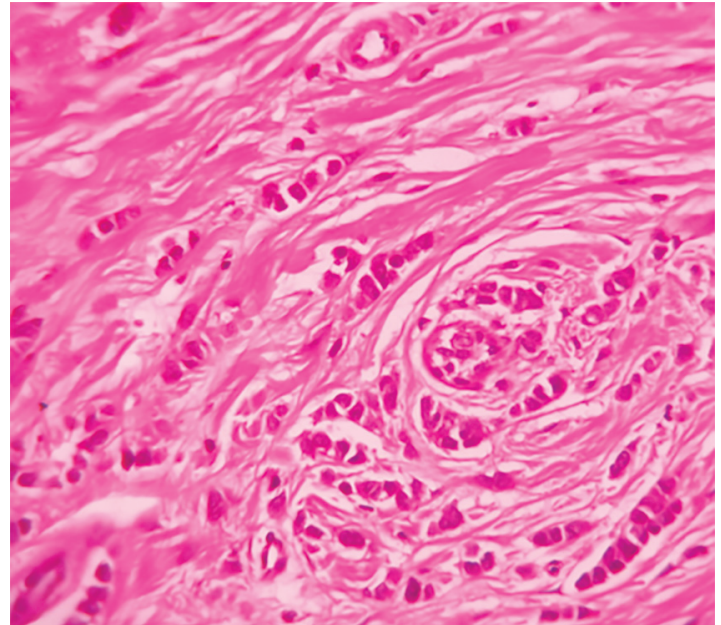

Fig. 14: Invasive lobular carcinoma, grade II (H and E 10X)

Table 2: Correlation between number of malignant lesions and CK 5/6 stain index positivity

\begin{tabular}{lll}
\hline Malignant & No. of cases & CK 5/6 stain index \\
\hline Idc (nos) & 35 & Grade III SI 2 \\
& & Rest SI 0 \\
Invasive papillary & 03 & SI-0 \\
Invasive lobular & 02 & SI-0 \\
Invasive mucinous & 01 & SI-0 \\
\hline
\end{tabular}

increasing tumor size. Tumor size and axillary lymph node status are highly correlated but are also independent measures of outcome. Survival declines with increasing tumor size when nodal status is held constant. ${ }^{13}$ A number of nodes involved, amount of carcinoma measured by the microscopic size of the largest nodal metastases and presence or absence of extranodal spread, are also prognostically important. ${ }^{14}$

In our study all benign breast lesions showed positive expression for CK $5 / 6$; the staining index of benign lesions varied from 5 to 9 . It correlated with other studies. Bhalla et al. reported positive immunoexpression in all benign cases with a staining index of 6-9 except in Lactating Adenoma. Akhtar et al. reported a stain index of $5-8 .{ }^{15}$ In the present study staining intensity and proportion of immunopositive cells was more in areas of hyperplasia, adenosis, and cystic change. Highest stain index was seen in fibrocystic with ductal epithelial hyperplasia. Most cases showed cytoplasmic and membranous staining. Most fibroadenomas showed a stain index of 6 . In benign phyllodes, there were areas of low cellularity showing cytoplasmic staining only with a stain index 5 .

In the present study 38 cases of infiltrating, carcinomas showed negative CK 5/6 expression. Only 3 cases showed weak positive expression. Findings correlated with other studies. Bhalla et al. reported in his study 6 out of 22 cases showed positive immunoreactions. Otterbach 
et al. also reported only 3 out of 39 infiltrating breast carcinomas showed CK 5/6 expression. ${ }^{16}$ Most malignancies are derived from differentiated glandular cells and do not reveal immunohistochemical staining with CK 5/6.

Lack of CK 5/6 expression in neoplastic cells of atypical hyperplasia, in-situ carcinomas and infiltrating carcinomas could be attributed to its arising from CK 5/6-negative glandular precursor cells. ${ }^{16}$ Also, the myoepithelial cell is absent in most invasive cancers helping in the differentiation of in situ carcinomas from invasive cancers. ${ }^{17}$ Lacroix et al reported that the expression rate of CK 5/6 in UDH, atypical ductal hyperplasia, IDC was $62.9 \%, 10.0 \%$ and 0 respectively. ${ }^{18}$ CK 5/6 was positive virtually in most benign ductal lesions and was expressed in glandular epithelium and myoepithelial cells. In DCIS, almost all the proliferating cells CK 5/6 was negative. In atypical ductal hyperplasia, only partially proliferating cells were positive. In invasive ductal carcinoma, no CK 5/6 was detectable with a complete lack of expression. Poorly differentiated carcinomas showed weak positive expression CK 5/6 because these high-grade IDC that are basal-like breast cancers possess an expression signature similar to basal/myoepithelial cells of the breast. $^{7}$

In our study, 28 cases were of size T2 $(>2 \mathrm{~cm}$ and $<5 \mathrm{~cm})$ and showed CK 5/6 stain index 0 . A $p$ value was insignificant. No consistent relation could be obtained. Herranz Mreported CK 5/6 positive IDCs had bigger tumor size $(p=0.005)$ and concluded that CK $5 / 6$ positivity was associated with lower age, greater size, distant metastases, grade 3, Ki-67 positive and $p 53$ positive. ${ }^{19}$ All these support associations between $\mathrm{CK} 5 / 6$ positivity and

Table 3: Supporting studies

\begin{tabular}{|c|c|c|c|}
\hline No. & Studies & $\begin{array}{l}\text { Correlation between } \\
\text { CK } 5 / 6 \text { positivity and } \\
\text { greater tumor size }\end{array}$ & $p$ value \\
\hline 1. & Rehim et al. ${ }^{20}$ & Positive & $p<0.001$ \\
\hline 2. & Bhalla et al. $^{7}$ & No consistent relation & $p>0.05$ \\
\hline 3. & Herranz ${ }^{19}$ & Positive & $p=0.005$ \\
\hline 4. & Akhtar et al. ${ }^{15}$ & No consistent relation & $p>0.05$ \\
\hline 5. & Present study & No consistent relation & $p=0.593$ \\
\hline
\end{tabular}

Table 4: Supporting studies

\begin{tabular}{llll}
\hline \multicolumn{5}{c}{$\begin{array}{l}\text { Correlation } \\
\text { between CK 5/6 } \\
\text { positivity and } \\
\text { No. }\end{array}$ Studies } & Grade III tumors & $p$-value \\
\hline 1. & Otterbach et al. $^{16}$ & Positive & - \\
2. & Rehim et al. $^{20}$ & Positive & $p<0.001$ \\
3. & Bhalla et al. $^{7}$ & Positive & $p<0.05$ \\
4. & Herranz $^{19}$ & Positive & $p=0:<0,0000$ \\
5. & Akhtar et al. $^{15}$ & Positive & $p<0.05$ \\
6. & Present study & Positive & $p$ value $=0.0005$ \\
\hline
\end{tabular}

basal-like tumors and it can define patient subgroups with different risks. Rehim et al. also reported a positive correlation between CK 5/6 positivity and greater tumor size $(p<0.001) .{ }^{21}$ Bhalla et al. $(p>0.05)$, Akhtar et al. $(p>0.05)$ found no consistent relation with tumor size and CK 5/6 positivity (Table 3 ).

All grade III infiltrating carcinoma showed weak positive immunohistochemical expression which correlated with other studies. Bhalla et al. reported all grade III malignant cases showed weak positive immunoreactions $(p<0.05)$. Akhtar et al. also reported all 6 cases of breast carcinoma positive for CK 5/6 expression were grade III and triple negative $(p<0.05)$. Rehim et al. reported a significant association between grade III carcinomas and CK 5/6 positivity $(p<0.001)$, he stated that invasive grade III carcinomas with extensive central necrosis had basal phenotype and aggressive behavior. But in low-grade invasive carcinoma myoepithelial layer is absent and so staining negative. Herranz reported CK 5/6 positive IDCs were more frequently grade III ( $p=0:<0,0000)$. CK 5/6 positivity in poorly differentiated carcinomas is because these high-grade IDC that are basal-like breast cancers possess an expression signature similar to basal/ myoepithelial cells of the breast ${ }^{7}$ (Table 4).

In the present study out of 7, only 3 cases with positive lymph node deposits showed stain index of 2 . The findings were consistent with other studies. Rehim et al. reported the inverse correlation between CK 5/6 positivity and nodal metastasis $(p=0.127)$. Akhtar et al. reported a positive correlation between CK 5/6 positivity and lymph node metastasis $(p<0.05)$. Dent et al. reported lymph node positivity in $55 \%$ cases of triple negative carcinomas while among other breast carcinomas $40 \%$ lymph node positivity. ${ }^{21}$ The larger sample size is required to establish conclusive results (Table 5).

\section{CONCLUSION}

With the current burden of breast neoplasm, the precision of diagnosis is of utmost importance to provide accurate treatment to patients. Immunohistochemistry (IHC) is an integral part of pathology. Although hematoxylin and eosin ( $\mathrm{H}$ and $\mathrm{E}$ ) stain remains gold standard method for

Table 5: Supporting studies

\begin{tabular}{llll}
\hline No. & Studies & $\begin{array}{l}\text { Correlation between } \\
\text { CK 5/6 positivity and } \\
\text { nodal metastasis }\end{array}$ & $p$ value \\
\hline & & & \\
1. & Rehim et al. $^{20}$ & Negative & $p=0.127$ \\
2. & Herranz $^{19}$ & No consistent relation & Not significant \\
3. & Akhtar et al. ${ }^{15}$ & Positive & $p<0.05$ \\
4. & Present study & Positive & $p=0.003$ \\
\hline
\end{tabular}


diagnosis, IHC provide vital information in differentiation in grey-zone cases.

CK 5/6, as a component of panels along with AE1/ AE3 and myoepithelial markers: smooth muscle actin, smooth muscle myosin heavy chain and p 63 help differentiate benign and malignant breast lesions in cases of interobserver variability. Grade III breast carcinoma cases, if positive for CK 5/6, imply a 'basal-like' molecular phenotype and signify a poor prognosis. These tumors require aggressive intervention. The patients with this subtype of breast cancer must be subjected to BRCA1 mutation testing. Thus with CK5/6, we can help to provide prognostic information and better treatment modalities.

\section{REFERENCES}

1. Hatim K, Laxmikant N, Mulla T. Patterns and prevalence of benign breast disease in western India. Int J Res Med Sci 2017; 5(2):684-688.

2. Sangma M, Panda K, Dasiah S. A clinico-pathological study on benign breast diseases. J Clin Diagn Res 2013;7(3):503-506.

3. Nandakumar A, Ramnath T, Chaturvedi M. The magnitude of cancer breast in India: A summary. Indian J Surg Oncol 2010;1:8-9.

4. Saranath D, Khanna A. Current status of cancer burden: Global and Indian scenario. Biomed Res J 2014; 1(1):1-5.

5. Babu GR, Lakshmi SB, Thiyagarajan AT. Epidemiological correlates of breast cancer in south India. Asian Pac J Cancer Prev 2013;14(9):5077-5083.

6. Yeh IT, Mies C. Application of immunohistochemistry to breast lesions. Arch Pathol Lab Med 2008;132:349-358.

7. Bhalla A, Manjari M, Kahlon K, Kumar P, Kalra N. Cytokeratin $5 / 6$ expression in benign and malignant breast lesions. Indian J Pathol Microbiol 2010; 53:676-680.

8. Foulkes WD, Stefansson IM, Chappuis PO, Begin LR, Goffin JR, Wong N, et al. Germline BRCA 1 mutations and a basal epithelial phenotype in breast cancer. J Natl Cancer Inst 2003; 95:1482-1485.

9. Kapoor S, Kumar A, Singh A, Singh H, Singla R. Varied pattern of breast diseases-A study of 443 Cases. IOSR J Dental and Medical Sci 2016;15(3):36-49.
10. Mudholkar GV, Mashal NS, Kawade BS. Histopathological study of neoplastic lesions of breast. Ind Med Gazette 2012; 45:118-122.

11. Ginsburg OM, Martin LJ, Boyd NF. Mammographic density, lobular involution, and risk of breast cancer. Br J Cancer 2008; 99:1369-1374.

12. Akhtar K, Bharduaj S, Naim M, Mansoor T, Sherwani R. Factors predicting the axillary lymph node metastasis in breast cancer: Is axillary node clearance indicated in every breast cancer patient? Ind J Surg 2011;73(5):331-335.

13. Carter C, Allen C, Henson D. Relation of tumor size, lymph node status, and survival in 24,740 breast cancer cases. Cancer 1989;63:181-187.

14. Ahmad Z, Khurshid A, Qureshi A, Idress R, Asghar N, Kayani $\mathrm{N}$. Breast carcinoma grading, estimation of tumor size, axillary lymph node status, staging, and Nottingham Prognostic Index scoring on mastectomy specimens. Ind J Pathol Microbiol 2009;52(4):477-481.

15. Akhtar K, Bharduaj S, Naim M, Mansoor T, Sherwani R. Diagnostic value of Cytokeratin 5 and Cytokeratin 6 in benign and malignant lesions of breast. Annuals of Pathology and Laboratory Med 2015;2(4):45-49.

16. Otterbach F, Bunkfalvi A, Bergner S, Decker T, Krech R, Boecker W. Cytokeratin 5/6 immunohistochemistry assists the differential diagnosis of atypical proliferations of the breast. Histopathol 2000;37:232-240.

17. Yanping D, Qiurong R. The value of p63 and CK5/6 expression in the differential diagnosis of ductal lesions of breast. J Huazhong Univ Med Sci 2006;26(4):405-407.

18. Lacroix-Triki M, Mery E, Voigt J. Value of cytokeratin 5/6 immunostaining using D5/16 B4 antibody in the spectrum of proliferative intraepithelial lesions of the breast: A comparative study with 34ßE12 antibody. Virchows Arch 2003; 442(6):548-554.

19. Herranz M. The expression of cytokeratin5/6 in Invasive Ductal Carcinoma of breast: Association with other clinical and biological parameters. Sci Stud Onc 2014;1:8-9.

20. Rehim D , Pinder SE, Paish CE, Bell J, Blamey RW, Robertson J. Expression of luminal and basal cytokeratins in human breast carcinoma. J Pathol 2004;203:661-671.

21. Dent R, Trudeau M, Pritchard KI, Hanna WM. Triple-negative breast cancer: clinical features and patterns of recurrence. Clin Cancer Res 2007;13(15):4429- 4434. 